\title{
A Novel High Dimensional and High Speed Data Streams Algorithm: HSDStream
}

\author{
Irshad Ahmed \\ Department of Computer Science \\ National University of Computer and \\ Emerging Sciences, Islamabad, Pakistan
}

\author{
Irfan Ahmed \\ Department of Computer Engineering \\ Taif University, Taif, KSA
}

\author{
Waseem Shahzad \\ Department of Computer Science \\ National University of Computer and \\ Emerging Sciences, Islamabad, Pakistan
}

\begin{abstract}
This paper presents a novel high speed clustering scheme for high-dimensional data stream. Data stream clustering has gained importance in different applications, for example, network monitoring, intrusion detection, and real-time sensing. High dimensional stream data is inherently more complex when used for clustering because the evolving nature of the stream data and high dimensionality make it non-trivial. In order to tackle this problem, projected subspace within the high dimensions and limited window sized data per unit of time are used for clustering purpose. We propose a High Speed and Dimensions data stream clustering scheme (HSDStream) which employs exponential moving averages to reduce the size of the memory and speed up the processing of projected subspace data stream. It works in three steps: i) initialization, ii) real-time maintenance of core and outlier micro-clusters, and iii) on-demand offline generation of the final clusters. The proposed algorithm is tested against high dimensional density-based projected clustering (HDDStream) for cluster purity, memory usage, and the cluster sensitivity. Experimental results are obtained for corrected KDD intrusion detection dataset. These results show that HSDStream outperforms the HDDStream in all performance metrics, especially, the memory usage and the processing speed.
\end{abstract}

Keywords-Evolving data stream; high dimensionality; projected clustering; density-based clustering; micro-clustering

\section{INTRODUCTION}

The exponential growth in data mining and clustering is an apparent result of the Internet penetration and the use of the network applications. Network applications have become an integral part of our daily life, whether it is related to the academic, research, health care, finance, business, or public service domains.

Data sources are monotonically increasing from past few decades. Additionally, the technological developments in data sensing systems (sensor networks) have resulted in a real-time data with large number of attributes. The large volume of the data together with its high dimensionality has motivated the research in the area of high dimensional data mining and exploration. Data stream is a form of data that continuously evolves reflecting the real-time variation in volume, dimensionality, and correlation. In recent years, a large amount of streaming data, such as network flows, wireless sensor networks data and the multimedia streams have been generated. Analyzing and mining of real-time streaming data have become a hot research topic [1], [2], [3]. Discovery of the patterns hidden in the streaming data imposes great challenges for cluster formation, especially in high dimensional data. By definition, a cluster is a collection of objects which are similar between them and are dissimilar to the objects belonging to other clusters. Data stream clustering algorithms are used to get important information from these streams in real-time. These algorithms search for the clusters that contain streaming objects with a certain degree of similarity across all dimensions. Stream clustering algorithms have special challenges that do not face most other clustering techniques. Storage and time limits are critical for clustering algorithms to perform a fast singlepass over that stream data. In addition to this, the evolving nature of the stream, requires the clustering algorithm to be highly adaptive to the new patterns. Generally, there are two types of stream clustering algorithms: full dimensional and projected or preferred dimension streaming algorithms. Clustering applications in various domains often have very highdimensional data; the dimension of the data being in the tens, hundreds or thousands, for example, in network streaming, web mining and bioinformatics, respectively. It is often require to focus on a certain subset of dimensions rather than the full dimension space because it requires less memory and render fast processing. In addition to the high dimensionality, realtime high-speed evolution makes it more intractable. Clustering such high-dimensional high-speed datasets is a contemporary challenge. Clustering algorithms must avoid the curse of dimensionality but at the same time should be computationally efficient. Some applications that generate data streams include: telecommunication (call records), network operation centers (log information from network entities), financial market (stock exchange), and day to day business (credit card, automated teller machine (ATM) transactions, etc). In a high dimensional dataset, among many features some attributes can be expected to be irrelevant for any given object of interest. Irrelevant attributes can obscure clusters that are clearly visible when we consider only the relevant subspace of the dataset. Therefore, clusters may be meaningfully defined by some of the available attributes only. The irrelevant attributes interfere with the efforts to find targeted clusters. This problem is become more intensive in streaming data, because it requires a single scan of the data to find the useful attributes for describing a potential cluster for the current object. Moreover, streams are impulsive and the discovered clusters might also evolve over time. High dimensional streaming data clustering is more challenging than the high density or high dimensional data. Among various challenges in clustering high dimensional streaming data [4], following two are the focuses of this paper: 
- Processing speed: Data streams arrive continuously, which requires fast and real-time response. The clustering algorithm needs to have processing speed (which comes from low complexity) such that it can handle the speed of data streams in the limited time.

- Memory usage: Large data streams are generated rapidly which need an unlimited memory. Therefore, the clustering algorithm must be optimized for realistic memory constraints.

In this paper, we introduce a novel tuple structure to summarize the high speed high dimensional data stream. This structure not only speed up the process but also requires less memory. Our clustering technique also modifies weights in some definitions of HDDStream, namely, the micro-cluster variance, projected dimensionality, projected distance, and projected radius. In terms of experimental results, we compare our scheme with HDDStream for cluster purity, memory usage, and cluster's sensitivity.

\section{Notations:}

Vectors and matrices are represented by bold letters, other notations are explained below:

$\mathbb{R}$ Set of real numbers

$\mathbb{N}$ Set of natural numbers

$\mathcal{C}$ Dataset

$\mathrm{N}$ Window size

$\epsilon \quad$ Radius threshold

$\mathcal{D}$ Dataset used in initialization phases

$\alpha \quad$ Exponential weighted average constant

$\beta \quad$ Outlier threshold

$\mu \quad$ Number of points threshold

$\xi \quad$ Variance threshold

$\psi_{j} \quad j^{\text {th }}$ preferred dimension

$\pi \quad$ Projected dimensionality threshold

\section{RELATED WORK}

In the last few years many research works have been done on high-dimensional data clustering and evolving data streams clustering. There are extensive research works on clustering algorithms for static datasets [5], [6], [4] where some of them have been further extended for evolving data streams. The clusters are formed based on a Euclidean distance function like $k$-means algorithm [7]. $k$-mean clustering splits the $n \mathrm{~d}$ dimensional points into $k$ cluster $(k<n)$. One of the wellknown extensions of $k$-means on data streams is presented by Aggarwal et al. [8]. They propose an algorithm called CluStream based on $k$-means for clustering evolving data streams. CluStream introduces an online-offline method for clustering data streams. CluStream clustering idea is adopted in the majority of data stream clustering algorithms. Aggarwal et al. extended their work in HPStream [9], which introduces the projected clustering to data streams. In projected clustering high dimensional stream data is partitioned based on the preferred dimensions instead of full the dimensional space. Cao et al. [10] use the density-based clustering without projected dimensions in DenStream algorithm. For streaming data, although a considerable research has tackled the fullspace clustering, relatively limited work deals with the subspace clustering. These few researches include [9] HPStream, [11] HDDStream, and [12] SubCMM. A more comprehensive review and classifications are given in survey [13]. In
[11], authors propose a density-based projected clustering scheme for high dimensional data streams called HDDStream. HDDStream works in three phases; an initial phase, in which initial set of core micro-clusters is formed, then online core and outlier clusters' maintenance with projected clustering, and finally, an on-demand offline clustering phase. Compared with the HPStream which requires the fixed number of clusters, the number of clusters in HDDStream is variably adjusted over time, and the clusters can be of arbitrary shape. SubCMM suggests a different way for evaluating stream subspace clustering algorithms by making use of available offline subspace clustering algorithms with the streaming environment to handle the errors caused by emerging, moving, or splitting subspace clusters. A recent, similarity-based Data Stream Classifier (SimC)[14] introduces an insertion/removal policy that adapts evolving data tendency and maintains a representative, small set of clusters. It uses instance based learning techniques to form adaptive clustering algorithm. In [1] clustering method based on a multi-agent system that uses a decentralized bottom-up self-organizing strategy to group similar data points is presented. It uses bio-inspired flocking model to eliminate the need of offline clustering. In [15], authors present a clustering algorithm for stream data with uncertain attributes has been presented in. This scheme works only for low dimensional streaming data. Liu [16] develop HSWStream algorithm. It is a data stream clustering algorithm based on exponential histogram over sliding windows with projected dimensions. Another density-based algorithm D-Stream [17] maps each input data into a grid, computes the density of each grid, and forms the clusters using these grids. In [18], authors propose a scalable algorithm to trace clusters in a high-dimensional data stream. The proposed scheme transforms the problem of multidimensional clustering into that of one-dimensional clustering along with a frequent item-set mining technique. This scheme achieves the scalability on the number of dimensions while sacrificing the accuracy of identified clusters. Bellas et al. [19] present an online variant of mixture of probabilistic principal component analyzers (MPPCA) to model and cluster the high dimensional high speed data. But to do so, it is necessary to add a classification step at the end of the online MPPCA algorithm to provide the expected clustering. MuDiStream [20] is a hybrid grid-based multi-density clustering algorithm with online-offline phases. In the online phase, it keeps summary information of evolving multi-density data stream in the form of core micro-clusters. The offline phase generates the final clusters using an adapted density-based clustering algorithm. The grid-based method is used as an outlier buffer to handle both noises and multi-density data in order to reduce the merging time of clustering. MuDi-Stream is not suitable for high-dimensional data since the number of empty grids increases which requires longer processing time. SE-Stream [21] is a standard-deviation based projected clustering method to support high dimensional data streams. It forms clusters within subgroups of dimensions and can detect change in the clustering structure during the progression of data streams. SED-Stream [22] is an extension of SE-Stream, in which some selected dimensions are used to represent the clusters to increase the quality of the output clustering. SEDStream projects any cluster to its discriminative dimensions that are highly relevant to the cluster itself but distinguished from the other clusters. SED-Stream is better than its previous version, SE-Stream, in terms of purity and f-measure. Both SE- 
Stream and SED-Stream use fading cluster structure (5-tuple) of the form similar to in section III definition 0 with two extra elements.

This paper presents High Speed and Dimensions data stream clustering scheme (HSDStream) which introduces a novel tuple structure to summarize the high speed high dimensional data stream. This structure not only speed up the process but also requires less memory. Our clustering technique also modifies weights in some definitions of HDDStream, namely, the micro-cluster variance, projected dimensionality, projected distance, and projected radius. In terms of experimental results, we compare our scheme with HDDStream for cluster purity, memory usage, and cluster's sensitivity.

\section{PROBLEM FORMULATION}

In general, data stream is modeled as an infinite series of points $\left\{\mathbf{p}_{1}, \mathbf{p}_{2}, \ldots, \mathbf{p}_{i}, \ldots\right\}$ arriving at discrete time $\left\{t_{1}, t_{2}, \ldots t_{i}, \ldots\right\}$. Each point $\mathbf{p}_{i}$ is a vector of dimension $d$ such that $\mathbf{p}_{i}=\left\{p_{i, 1}, p_{i, 2}, \ldots, p_{i, d}\right\}$.

An important characteristic of data streams is that we cannot store all data points. A usual way to overcome this problem is to summarize the data through an appropriate summary structure, often called micro-cluster. A micro-cluster summarizes the time and dimensionality limited stream data in the form of a tuple. When aging is also under consideration, the temporal extension of micro clusters [9] is employed. Recent research works [9], [11] use the following definition of microcluster:

Definition 0. (Micro-cluster $m c$ )

A micro-cluster at time $t$ for a set of d-dimensional data points $\mathcal{C}=\left\{\mathbf{p}_{0}, \mathbf{p}_{1}, \ldots, \mathbf{p}_{N-1}\right\}$ arriving at discrete time $t_{0}, t_{1}, \ldots, t_{N-1}$, is summarized as $(2 d+1)$ size tuple $m c(t)=$ $\{$ CF1 $(t), \mathbf{C F} 2(t), W(t)\}$, where CF1 $(t)$ and CF2 $(t)$ are $d$ dimensional vectors, defined as:

- $\mathbf{C F 1}(t)$ is the d-dimensional vector of weighted sum of points $\left\{\mathbf{p}_{1}, \mathbf{p}_{2}, \ldots, \mathbf{p}_{i}, \ldots\right\}$ along each dimension, such that for dimension $j$ we have $C F 1_{j}=$ $\sum_{i=0}^{N-1} p_{i, j} f\left(t-t_{i}\right)$, where $N$ is the size of time window, $p_{i, j}$ is the $i^{t h}$ point in time window and $f\left(t-t_{i}\right)$ is the weight of the $i^{t h}$ point.

- $\quad$ CF2 $(t)$ is the d-dimensional vector of weighted sum of the squares of the points $\left\{\mathbf{p}_{1}, \mathbf{p}_{2}, \ldots, \mathbf{p}_{i}, \ldots\right\}$ along each dimension, such that for dimension $j$ we have $C F 2_{j}=\sum_{i=0}^{N-1} p_{i, j}^{2} f\left(t-t_{i}\right)$, where $N$ is the size of time window, $p_{i, j}$ is the $i^{t h}$ point in time window and $f\left(t-t_{i}\right)$ is the weight of the $i^{t h}$ point.

- $W(t)$ is the sum of the weights of data points, mathematically, $W(t)=\sum_{i=0}^{N-1} f\left(t-t_{i}\right)$.

In data streams, since we are more interested in the data within a certain recent time window instead of all historical data, an aging effect has been used for weighted function $W(t)$. Recent works [9], [11] have used conventional exponential fading function $f(t)=2^{-\lambda t}$, where $\lambda$ is the decay rate. By using fading function $f(t)$ we need to maintain a memory buffer of time window size for each cluster, because, whenever a new point arrives we need to shift the previous data in the buffer of fixed size. We want to highlight an important

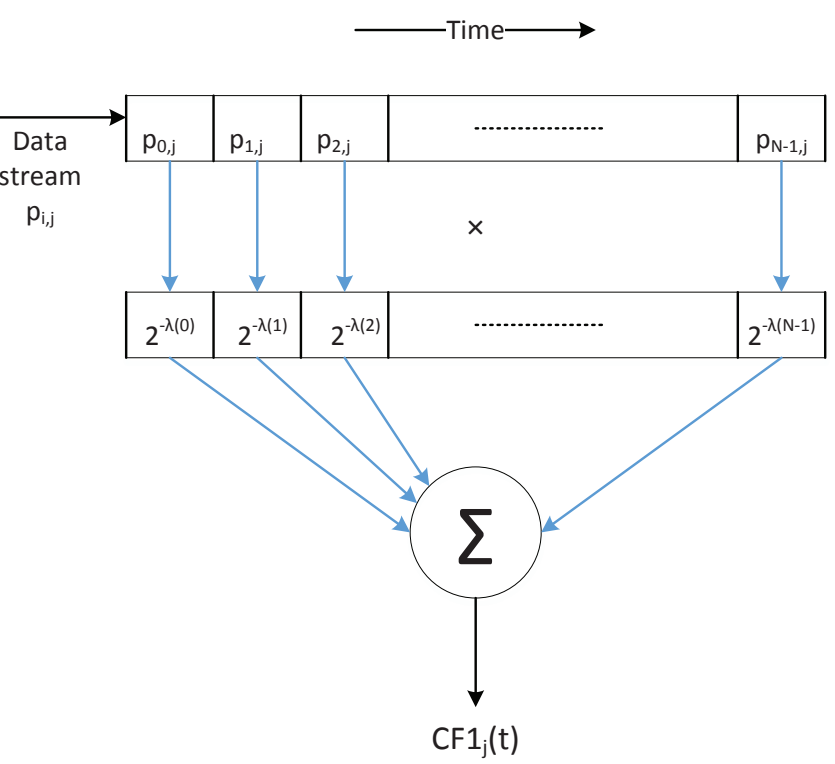

Fig. 1. Practical approach to update micro-cluster tuple

point here that the online update of the tuples [9], [11] of the form $m c(t)=\left\{\mathbf{C F 1}(t)+p, \mathbf{C F 2}(t)+p^{2}, W(t)+1\right\}$ is not practically feasible because it leads to monotonically increasing weighted sum data. A practical approach for updating the tuple is shown in Fig. 1. It is obvious that for a fixed size memory shift register, when a new point arrives the old point is discarded. The correct mathematical expression for online update, then, becomes, $m c(t)=\left\{\mathbf{C F} \mathbf{1}(t)-\mathbf{C F} \mathbf{1}_{N-1}+\right.$ $\left.p, \mathbf{C F} 2(t)-\mathbf{C F} 2_{N-1}+p^{2}, W(t)-f\left(t-t_{N-1}\right)+1\right\}$, such that for dimension $j$ we have $C F 1_{N-1, j}=p_{N-1, j} f\left(t-t_{N-1}\right)$ and $C F 2_{N-1, j}=p_{N-1, j}^{2} f\left(t-t_{N-1}\right)$.

We define micro-cluster as follows:

Definition 1. (Micro-cluster $m c$ ) We redefine the micro-cluster as a set of points $\mathcal{C}=\left\{\mathbf{p}_{0}, \mathbf{p}_{1}, \ldots, \mathbf{p}_{N-1}\right\}$ arriving at discrete time points $t_{0}, t_{1}, \ldots, t_{N-1}$. The $m c$ is summarized as $(2 d+1)$ size tuple $m c(t)=\{\mathbf{E A} \mathbf{1}(t), \mathbf{E A} 2(t), W(t)\}$, where $\mathbf{E A} \mathbf{1}(t)$ and $\mathbf{E A 2}(t)$ are $d$ dimensional vectors, defined as:

- $\mathbf{E A 1}(t)$ is the d-dimensional vector of exponential weighted moving average of points $\left\{\mathbf{p}_{1}, \mathbf{p}_{2}, \ldots, \mathbf{p}_{i}, \ldots\right\}$ along each dimension, such that for dimension $j$ we have $E A 1_{j}(t)=\alpha p_{j}(t)+(1-\alpha) E A 1_{j}(t-1)$, where $\alpha=2 /(1+N)$ is a smoothing factor controlled by the size of time window; and $p_{j}(t)$ is the latest point in time window.

- $\mathbf{E A 2}(t)$ is the d-dimensional vector of exponential weighted average of points $\left\{\mathbf{p}_{1}, \mathbf{p}_{2}, \ldots, \mathbf{p}_{i}, \ldots\right\}$ along each dimension, such that for dimension $j$ we have $E A 2_{j}(t)=\alpha p_{j}^{2}(t)+(1-\alpha) E A 2_{j}(t-1)$.

- $W(t)$ is the sum of the of data points at time $t$.

In order to formalize aging effect of data we introduce exponential moving average of data stream within a specified 


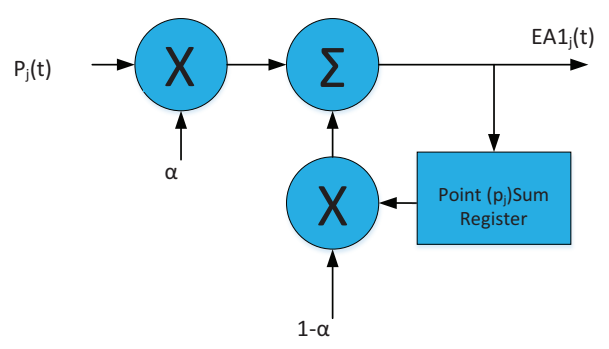

Fig. 2. Exponential moving average based update of micro-cluster tuple

time window. We use exponential weighted moving average in the tuple as decreasing exponential function. Note that, now the calculation of $\mathbf{E A 1}(t)$ or $\mathbf{E A 2}(t)$ does not require storage of past values, and only one addition and two multiplications with one memory register (of the size of dimension $j$ ) are required to update the tuple at any time instance. Design implementation of our micro-cluster update is shown in Fig. 2.

Data stream contains high dimensional data where each dimension has its own importance. In order to collate the similar points in data stream we use variance along each dimension. The lower the variance the higher the correlation among the points in particular dimension. We use variance as a metric to limit the number of dimensions to preferred dimensions only.

Definition 2. (Preferred Dimension) A dimension $j$ is said to be a preferred dimension if $\operatorname{Var}_{j}(m c)<\xi$, where $\xi$ is the variance threshold and $\operatorname{Var}_{j}(m c)$ is the variance of $m c$ along dimension $j$, defined as:

$$
\operatorname{Var}_{j}(m c)=E A 2_{j}(t)-\left(E A 1_{j}(t)\right)^{2}
$$

The preferred dimension helps gather the data points which have preferred dimensions less than a pre-defined threshold. Intuitively, it indicates the similarity across dimensions controlled by the variance threshold $(\xi)$. In conjunction with preferred dimension, we define the preferred dimension vector.

Definition 3. (Preferred Dimension Vector) Every microcluster has a preferred dimension vector defined as:

$$
\Psi(m c)=\left\{\psi_{1}, \psi_{2}, \ldots, \psi_{d}\right\}
$$

with

$$
\psi_{j}= \begin{cases}\varrho, & \operatorname{Var}_{j}(m c)<\xi \\ 1, & \text { otherwise. }\end{cases}
$$

where $\xi \in \mathbb{R}$, and $\varrho \in \mathbb{R}$ is a constant $\varrho \gg 1$. The number of elements in preferred dimension vector gives the projected dimensionality of the micro-cluster. The term 'projected' differentiates the micro-cluster defined over s projected subspace of the feature space instead of the whole feature space.

Definition 4. (Projected Dimensionality) Let $p \in \mathcal{C}$ and $\xi \in \mathbb{R}$. The number of dimensions $j$ with $\operatorname{Var}_{j}(m c)<\xi$ is called projected dimensionality of $m c$ and denoted by $\operatorname{PDIM}(m c)$.

Weighting the dimensions inversely proportional to their variance is not useful because we are only interested in distinguishing between dimensions with low variance and all other dimensions. Therefore, we use only two-valued weight vector. It can be easily determined from the preferred dimension vector by counting the number of dimensions with the normalization factor $\varrho$. The intuition of calculating projected dimensionality is to find projected core micro-cluster, i.e., the clusters with some subspace of dimensions instead of all dimensions.

Definition 5. (Projected Radius) Let $m c$ be a micro-cluster, $\xi \in \mathbb{R}$, and $\varrho \in \mathbb{R}$ is a constant $\varrho \gg 1$. The projected radius of $m c$ is given by:

$$
r_{\Psi}(m c)=\sqrt{\sum_{j=1}^{d} \frac{\psi_{j}}{\varrho}\left(E A 2_{j}(t)-\left(E A 1_{j}(t)\right)^{2}\right)}
$$

where $\varrho$ normalizes the variance along each dimension. This is the projected radius that takes into account the preferred dimensions of the micro-cluster.

Definition 6. (Projected Distance) Let $p \in \mathcal{D}$ and $m c$ be a projected micro-cluster with dimension preference vector $\Psi(m c)$. The projected distance between $p$ and $m c$ is given by:

$$
d i s t^{p r o j}(p, m c)=\sqrt{\sum_{j=1}^{d} \frac{\psi_{j}}{\xi}\left(p_{j}-\text { center }_{j}^{m c}\right)^{2}}
$$

where center ${ }^{m c}$ is the center of micro-cluster $m c$ and is given by center $^{m c}=\mathbf{E A} \mathbf{1}(t)$.

Now we introduce the notion of core-projected $m c$ which is an essential component of density based clustering. A coreprojected $m c$ is a $m c$ that contains at least $\mu$ number of points within a projected radius of $\epsilon$ with projected dimensionality less than a threshold $\pi$.

Definition 7. (Core Projected Micro-cluster) Let $\epsilon, \xi \in \mathbb{R}$ and $\pi, \mu \in \mathbb{N}$. A micro-cluster $m c$ is called a core projected $m c$ if the preference dimensionality of $m c$ is at most $\pi$ and it contains at least $\mu$ points within its projected radius $\epsilon$, formally:

$$
\begin{aligned}
& \operatorname{CORE}^{p r o j}(m c) \Longleftrightarrow \\
& \left(r_{\Psi}(m c)<\epsilon\right) \wedge(W(t)>\mu) \wedge(\operatorname{PDIM}<\pi) .
\end{aligned}
$$

In other words, a micro-cluster $m c$ is a core projected $m c$ iff:

(1) $r_{\Psi}(m c)<\epsilon$

(2) $W(t)>\mu$

(3) PDIM $<\pi$

There might be micro-clusters that do not fulfill the above constraints either because their associated number of points is smaller than $\mu$ or because their projected dimensionality exceeds $\pi$. These micro-clusters are treated as outliers.

Definition 8. (Outlier Micro-cluster) Let $\epsilon, \xi \in \mathbb{R}$ and $\pi, \mu \in$ $\mathbb{N}$. A micro-cluster $m c$ is called a outlier $m c$, if its projected dimensionality is at least $\pi$ and its projected radius and $\epsilon$ Neighbors are at most $\epsilon$ and $\mu$, respectively, formally:

$$
\begin{aligned}
& \operatorname{outlier}(m c) \Longleftrightarrow \\
& (\operatorname{PDIM}>\pi) \wedge\left(r_{\Psi}(m c)<\epsilon\right) \wedge(W(t)<\mu) .
\end{aligned}
$$


In order to keep update the micro-clusters, i.e., to check for possible conversion of core micro-cluster to outlier microcluster and vice versa, we introduce an outlier threshold $(0<$ $\beta<1$ ) such that an outlier micro-cluster becomes a potential core micro-cluster if $W>\beta \mu$ in addition to the conditions in (6) . Similarly, a core micro-cluster becomes a potential outlier micro-cluster if $W<\beta \mu$ in addition to the conditions in (7). The micro-cluster can be easily maintained online when a new point arrives in a cluster and other $m c$ need time degradation.

Remark. (Online maintenance) The micro-cluster $m c$ defined in definition 1 holds simple additive property that facilitates the online maintenance.

- If a point $p$ arrives at time $t$, then the updated tuple is given by $m c(t)=\left\{\alpha p+(1-\alpha) E A 1(t-1), \alpha p^{2}+\right.$ $(1-\alpha) E A 2(t-1), W(t-1)+1\}$.

- If no point adds in a micro-cluster at time $t$, then the updated tuple is given by $m c(t)=\{(1-\alpha) E A 1(t-$ 1), $(1-\alpha) E A 2(t-1), W(t-1)\}$.

\section{The HSDStream Algorithm}

HSDStream algorithm can be divided into three parts: 1) initialization to produce a set of representative core microcluster (core-mc) from an initial chunk of data points, 2) online maintenance of core-mc and outlier micro-cluster (outlier-mc), and, 3 ) the on-demand offline generation of the final clusters.

\section{A. Initialization}

In order to get initial set of micro-clusters from a fixed size of data points, we apply density-based projected clustering algorithm, a variant of PreDeCon algorithm [23], which is designed to work for fixed size of high dimensional data. Let $\mathcal{D}$ be a set of initial chunk of d-dimensional data points $\left(\mathcal{D} \subseteq \mathbb{R}^{d}\right)$. For each point $p \in \mathcal{D}$, we find a set of $\epsilon$-neighbors $\mathcal{N}_{\epsilon}(p)$, where $\epsilon$ is the radius threshold. In addition to this, we find the neighbors of $p$ with projected distance equal to or less than the $\epsilon$, namely, $\mathcal{N}_{\epsilon}^{\Psi(p)}(p)$.

Definition 9. (Projected Distance of a Point) Let $p, q \in \mathcal{D}$. The projected distance of a point $p$ with any point $q$ is given by:

$$
\operatorname{dist}_{p}(p, q)=\sqrt{\sum_{i=1}^{d} \frac{\psi_{i}(p)}{\varrho}\left(d_{i}(p)-d_{i}(q)\right)^{2}}
$$

where $d_{i}(p)$ is the $i^{t h}$ dimension of point $p$. Note that, in general $\operatorname{dist}_{p}(p, q) \neq \operatorname{dist}_{p}(q, p)$ because of the projected dimension vectors of point $p$ and $q$. In order to get symmetrical distance between $p$ and $q$ we use maximum of $\operatorname{dist}_{p}(p, q)$ and $\operatorname{dist}_{p}(q, p)$.

A projected core point $o \in \mathcal{D}$ can be defined with the same intuition of projected micro-cluster in definition 7 .

$$
\operatorname{CORE}^{p r o j}(o) \Longleftrightarrow \operatorname{PDIM}\left(\mathcal{N}_{\epsilon}(o)\right) \leq \pi \wedge\left|\mathcal{N}_{\epsilon}^{\Psi(p)}(o)\right| \geq \mu
$$

The initialization function in algorithm 1 line 5 runs the algorithm for the creation of initial set of $m c$. It starts by inserting all points in the set $\mathcal{N}_{\epsilon}(o)$ into a queue. For each point in the queue, it computes all directly projected weighted reachable points and inserts those points into the queue which

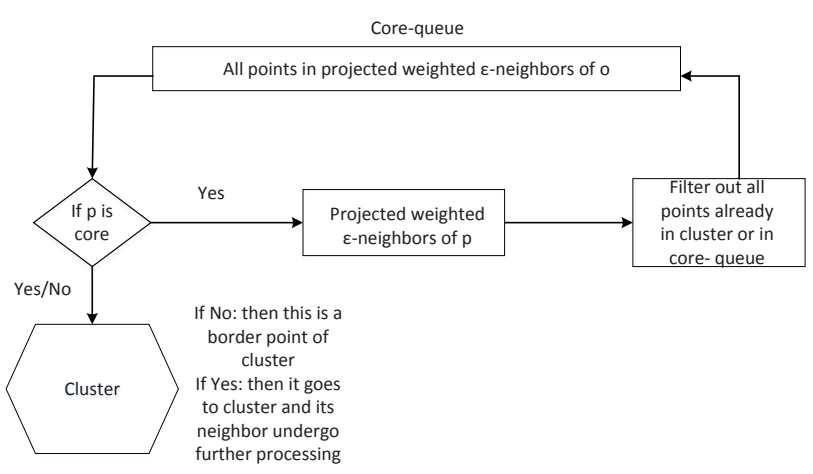

Fig. 3. Generation of initial set of micro-clusters

are still unclassified. This process repeats until the queue is empty and the cluster is computed. The flow chart of algorithm is shown in Fig. 3. Remove all those points belong to calculated cluster from dataset $\mathcal{D}$ and repeat the process for another core point. This process remains continue till all the core points are exhausted.

\section{B. Real-time Maintenance of Micro-clusters}

In order to find out the clusters in an evolving real-time data stream, we maintain two groups of micro-clusters, namely, core-mc and outlier-mc in real-time. All the micro-clusters are maintained in a separate memory space. A new point might be assigned to core-mc, outlier-mc, or it may start new outlier-mc depends upon various factor. Sequential process of merging a new point $p$ is described below:

1) When a new point arrives, it first becomes the candidate of core-mc (algorithm 1, line 13). The projected dimensionality of each core-mc has been evaluated before and after adding this point $p$ (algorithm 2, line 4). After that, projected distance of $p$ is calculated with those core-mc which still satisfy the projected dimensionality constraint, i.e., after the addition of point $p$ (algorithm 2, line 6). Then, we choose one core-mc which has smallest projected distance from $p$ (algorithm 2, line 9). Finally, the projected radius of chosen core-mc ( $p$ included) has been evaluated and checked for upper bound $(\epsilon)$ (algorithm 2, line 11). If it satisfies, then point $p$ is assigned to that core-mc (algorithm 2, line 12 using update tuple function in algorithm 4), else it becomes candidate of outlier-mc list.

2) When a new point becomes a candidate for an outliermc, the projected distance of $p$ with each outlier-mc is evaluated (algorithm 3, line 4). The closest distant outliermc is chosen in line 6 . The point $p$ becomes the member of that outlier-mc if the projected radius is less than or equal to the radius threshold $(\epsilon)$ (algorithm 3, line 9). In order to get long term effect we check the possibility of outlier-mc to core-mc conversion after certain number of points (window size $N$ ).

3 ) If point $p$ cannot be added in core-mc or outlier-mc (algorithm 3, line 14) then a new outlier-mc is created with this point being the first element. It may become the seed of future core-mc. 

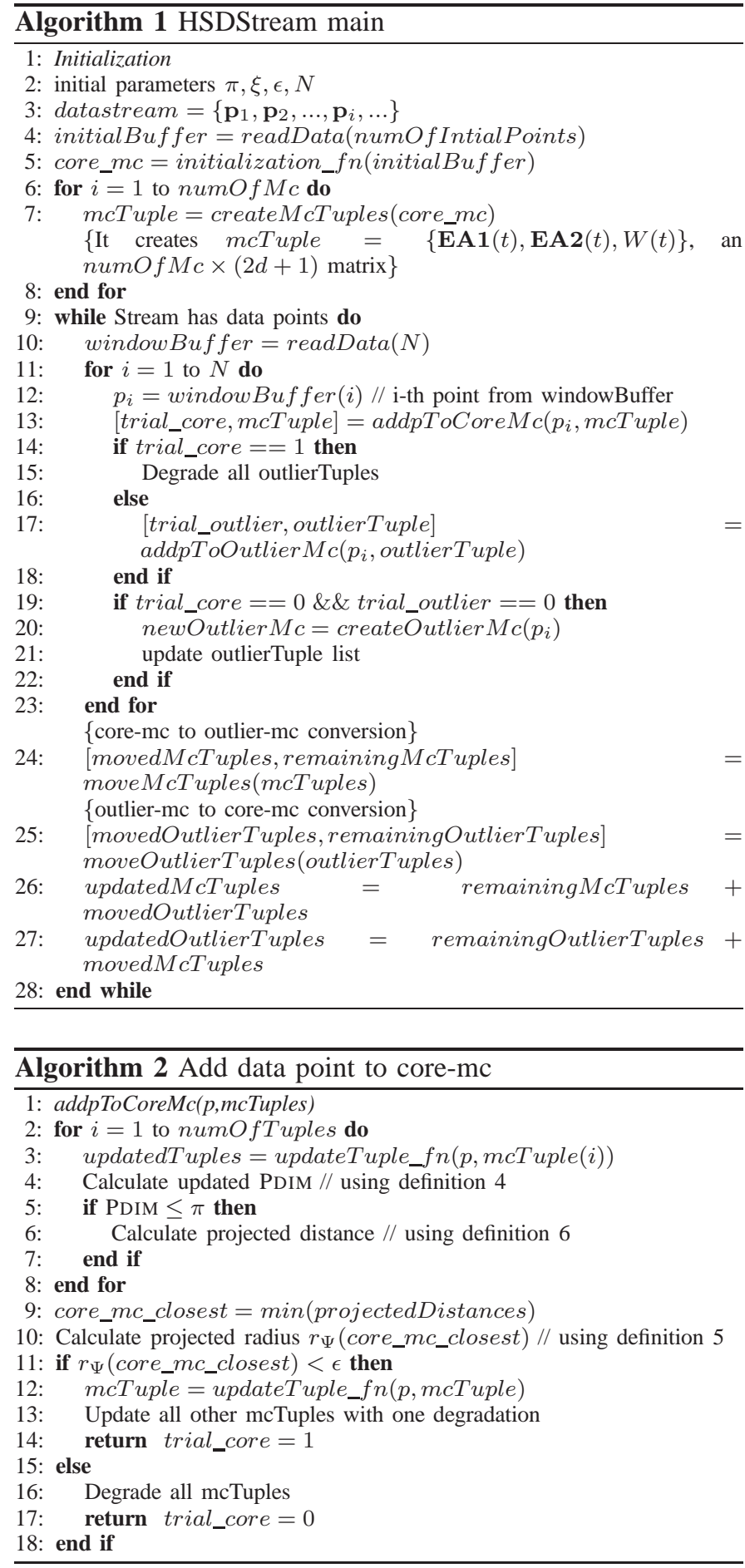

\section{Clusters Generation: Offline}

The real-time maintained micro-clusters capture the density area and the projected dimensionality of data streams. However, in order to get meaningful clusters, we need to apply some clustering algorithm to get the final result. When a clustering request arrives, a variant of PreDeCon algorithm [23] is applied on the set of real-time maintained core-mc(s) to get the final result of clustering. In density-based PreDeCon, a core point starts a micro-cluster, all the directly connected points and the chain of core points which satisfy $\epsilon$-neighborhood
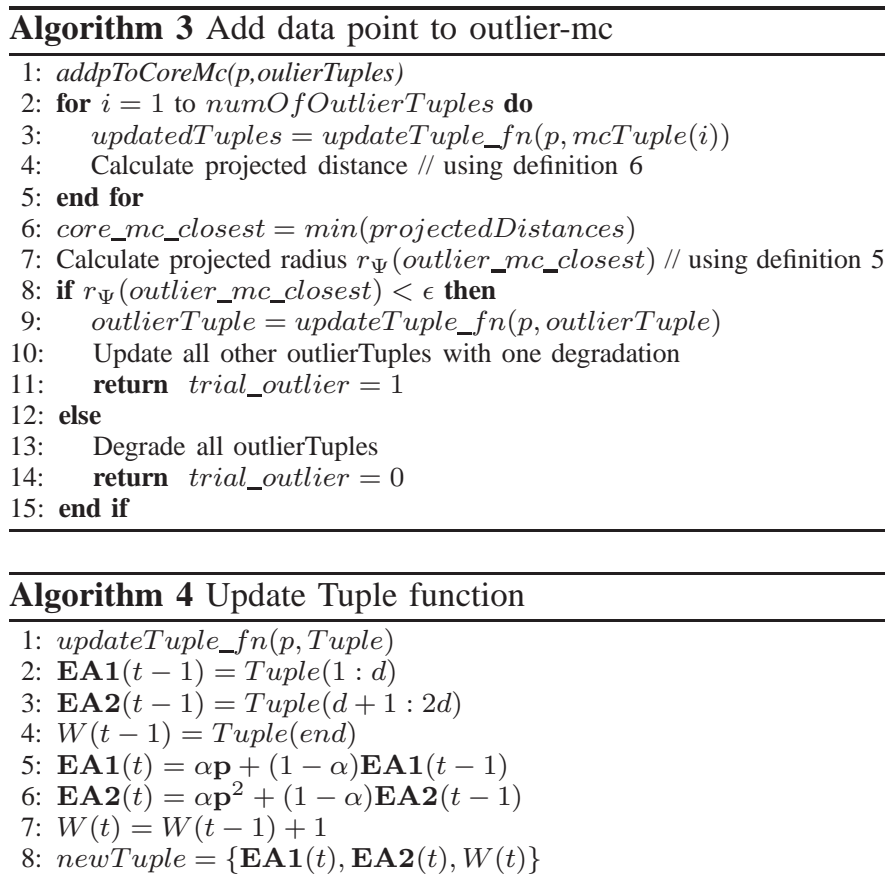

criteria and maximum dimensionality $\pi$ become the member of that cluster. During offline on-demand clustering phase, each core-mc acts as core point. Each core-mc is regarded as a virtual point located at the center of core-mc. We use the concept of density connectivity to determine the final clusters, i.e., all the density-connected core-mc(s) form a cluster.

\section{DISCUSSION}

In this section we highlight issues and challenges in the development of high dimensional data stream clustering in Internet traffic monitoring. We maintain the density with $\epsilon-$ neighborhood and minimum number of points $\mu$ in a coremc. When an identical burst of data (in case of attack on network) arrives, outlier-mc(s) are diminished and only one core-mc remains there. In this case, an important entity of core-mc formation i.e., projected dimensionality cannot work because, now PDIM $=d$ and it no longer satisfies the condition PDIM $\leq \pi$. In order to overcome this problem we introduce another condition ORed with the condition PDIM $\leq \pi$ to maintain one core-mc containing exactly similar data. The new condition is $W(t) / N>90 \%$, i.e., if the data points window contains more than $90 \%$ points, then no need to check PDIM because the majority of identical data points indicates some abnormal activity on the network being monitored. During real-time maintenance, when a new point arrives and it becomes a part of only one micro-cluster, then, all the other micro-clusters undergo one time degradation. For each existing core-mc, if no new point is merged into it, then the weight of core-mc will decay gradually. If the weight is below $\beta \mu$, then it means that core-mc has become an outlier-mc, it should be deleted and its memory space should be released for new coremc. Similarly, if the weight is above $\beta \mu$ then it means that the outlier-mc has become a core-mc, it should be deleted and its memory space should be released. Therefore, we need to check the weight of each micro-cluster periodically. We use a fixed time period to perform this check at every time window 
interval $(N)$. In this way any outlier-mc automatically vanishes if no point merges in it during $N$ time units.

\section{EXPERIMENTAL EVALUATION}

We compare our proposed HSDStream algorithm with HDDStream [11] which is the recent projected clustering algorithm for high dimensional data streams. We use corrected KDD (Knowledge Discovery and Data mining) 1999 [24] Computer Network Intrusion detection dataset which is typically used for the evaluation of stream clustering algorithms. Both algorithms are implemented in MATLAB and run on Intel i5 Dual Core 2.0GHz with 2 GB RAM.

\section{A. Dataset}

To evaluate the performance of clustering algorithm we use KDD 1999 Network Intrusion detection dataset. This is the dataset used for The Third International Knowledge Discovery and Data Mining Tools Competition, which was held in conjunction with KDD-99 The Fifth International Conference on Knowledge Discovery and Data Mining. It has been reported that original dataset contains bugs, therefore, we use the corrected dataset available online at http://kdd. ics.uci.edu/databases/kddcup99/kddcup99.html. KDD-CUP'99 Network Intrusion Detection stream dataset which has been used earlier [8], [9], [10], [11] to evaluate CluSTREAM, HPStream, DenStream, HDDStream, respectively. This dataset corresponds to the important problem of automatic and realtime detection of network attacks and consists of a series of transmission control protocol (TCP) connection records from two weeks of local area network (LAN) traffic managed by MIT Lincoln Labs. Each record can either corresponds to a normal connection, or an intrusion. Most of the connections in this data set are normal, but occasionally there could be a burst of attacks at certain times. In this dataset, attacks fall into four main categories:

- DOS: denial-of-service e.g., syn flood

- R2L: unauthorized access from a remote machine, e.g., guessing password

- U2R: unauthorized access to local superuser (root) privileges, e.g., various "buffer overflow" attacks

- Probing: surveillance and other probing, e.g., port scanning

The attack-types are further classified into one of 24 types, such as back, buffer_overflow, ftp_write, guess_passwd, imap, ipsweep, spy, and so on. It is obvious that each specific attack type can be treated as a sub-cluster. Also, this data set contains totally 494020 connection records, and each connection record has 42 attributes or dimensions that belongs to one of the continuous (35) or symbolic type (7). In the performance analysis of proposed algorithm we use all 35 continuous attributes.

\section{B. Cluster Quality Evaluation}

Traditional full dimensional clustering algorithms, for example, [8] used the sum of square distances (SSQ) to evaluate the clustering quality. However, SSQ is not a good measure in evaluating projected clustering [9] because it is a full
TABLE I. PARAMETER VALUES

\begin{tabular}{cc}
\hline Parameter & Value \\
\hline$N$ & 200 \\
$\pi$ & 30 \\
$\mu$ & 10 \\
$\beta$ & 0.2 \\
$\xi$ & 0.002 \\
initialPoints & 1000 \\
$\epsilon$ & 10 \\
$H$ & 1 \\
\hline
\end{tabular}

dimensional measure, and full dimensional measures are not very useful for measuring the quality of a projected clustering algorithm. So, as in [9] and [11], we evaluate the clustering quality by the average purity of clusters, which examines the purity of the clusters with respect to the true cluster (class) labels. The purity is defined as the average percentage of the dominant class label in each cluster [10]. Let there are $K$ number of cluster in a cluster set $\mathcal{K}$ at query time such that $k \in \mathcal{K}=\{1,2, \ldots, K\}$.

$$
\operatorname{purity}(\mathcal{K})=\frac{\sum_{k=1}^{K} \frac{\left|P_{k}^{d}\right|}{\left|P_{k}\right|}}{K}
$$

where $\left|P_{k}^{d}\right|$ is the number of points with dominant class label in cluster $k$ and $\left|P_{k}\right|$ is the number of points in cluster $k$. Intuition behind the cluster purity is to measure the actual capture of distinct groups of data points which are known to the given dataset. The time span in which we measure the purity is called Horizon window $H$. It is measured in the number of time windows $N$. In the performance analysis $H=1$ otherwise stated.

Fig. 4-8 show the cluster purity of HDDStream and HSDStream. In network streaming data, normal traffic packets (or points) are random in nature at any particular time interval, however, a network attack is characterized by bursts of correlated data packets. Therefore, we cannot fit normal traffic packets in a single cluster. We can fine tune the design parameters $(\alpha, \beta, \xi, N)$ to capture the known types of attacks or even the unknown abnormal traffic patterns. We can see that cluster purity can take values from 0 to 1 . Cluster purity for normal network traffic usually varies from 0.5 to 1 . It can go below 0.5 if we have more than $50 \%$ data points with more than $20 \%$ dimensions outside the standard deviation of cluster in a certain time window. Intuitively, cluster purity is low if the cluster contains uncorrelated data or in other words, the normal data traffic. High purity (or purity 1) corresponds to highly correlated data as a result of some network attack. In Fig. 4, smurf attack can be seen between $34-57$ time units (for $N=200$ ) which corresponds to data points 7795 to 11489 in the KDD network intrusion database. The network is again under smurf attack from 211 to 249 time units. During the time interval from 250 to 365 we encounter with several attacks (back, ipsweep, nmap, and neptune) along with correlated normal data so that we can see cluster purity is equal to 1 for this time interval. Satan attacks the network from 453 to 455 time units, followed by smurf attack which continues till the end of simulations at 495 time units. It can be observed that HDDStream has the same purity graph pattern as HSDStream but with considerably low magnitude. This is due to the large number of core-mc(s) in HDDStream and the fact that percentage purity is inversely proportional to the number of clusters (10). The average cluster purity for HSDStream 


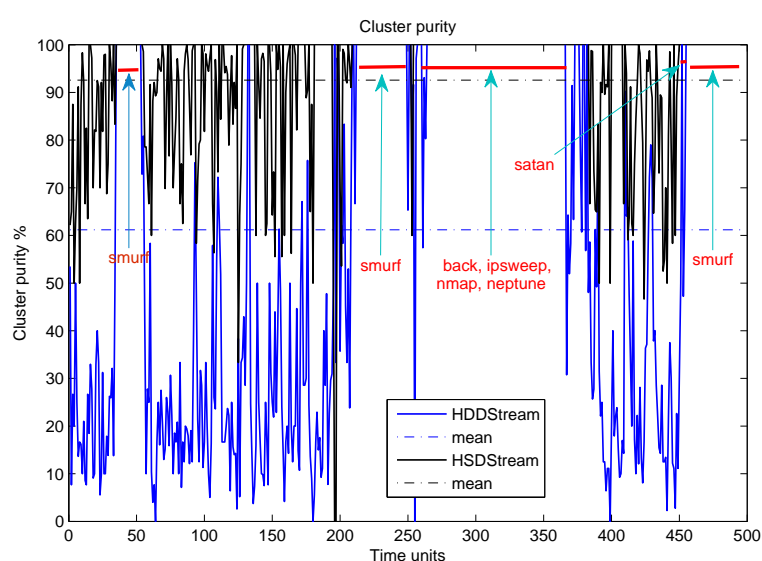

Fig. 4. Cluster purity with default values of parameters

is $92.57 \%$ as compared to the $61.18 \%$ of HDDStream. Next we illustrate: Why HSDStream has fewer number of clusters compared to HDDStream. Since the velocity of points is same for both schemes, it implies that HSDStream has more points per cluster than the HDDStream. For HSDStream, mean value of points in a window $N$ is given by

$$
\begin{aligned}
\mathbf{E A 1}(n)= & \alpha(1-\alpha) \mathbf{p}_{n}^{n-n}+\alpha(1-\alpha)^{n-\overline{n-1}} \mathbf{p}_{n-1} \\
& +\alpha(1-\alpha)^{n-\overline{n-2}} \mathbf{p}_{n-2}+\ldots+\alpha(1-\alpha)^{n} \mathbf{p}_{0}
\end{aligned}
$$

where $\alpha=2 /(1+N)$. Let $N=200$ and $n=\{0,1,2, \ldots 199\}$ with 0 being the first point and 199 is the latest point in a buffer window. Similarly, the mean value of points in window $N$ is given by

$$
\begin{aligned}
\frac{\mathbf{C F} 1(n)}{W}= & \frac{2^{-\lambda\left(\frac{n-n}{N}\right)}}{W} \mathbf{p}_{n}+\frac{2^{-\lambda\left(\frac{n-\overline{n-1}}{N}\right)}}{W} \mathbf{p}_{n-1} \\
& +\frac{2^{-\lambda(n-\overline{n-2})}}{W} \mathbf{p}_{n-2}+\ldots+\frac{2^{-\lambda\left(\frac{n}{N}\right)}}{W} p_{0}
\end{aligned}
$$

Substituting the values of parameters, we get EA1(199) = $0.01 p_{199}+0.009 p_{198}+0.0098 p_{197}+\ldots+0.0014 p_{0}$ and CF1 $/ W=0.0054 p_{199}+0.0054 p_{198}+0.0054 p_{197}+\ldots+$ $0.0046 p_{0}$. Thus, for the same point HSDStream gives larger mean value than HDDStream. From equation (5), it is obvious that higher values of mean (center) result in smaller projected distance, hence larger number of points per cluster and fewer number of clusters.

Fig. 5 depicts the cluster purity for default values of parameters in bar graph. It can be noticed that HSDStream and HDDStream are equally good in detecting the attacked points but the cluster purity for normal traffic is low in HDDStream because of large number of clusters (low density clusters). Fig. 6 shows the cluster purity with $N=100$. By decreasing the window size we actually increase the granularity and can capture smaller attacks. The price for this granularity is the more processing for the same amount of data. Again the average value of cluster purity for HSDStream is significantly larger than the HDDStream: 95.23 versus 67.31. Fig. 7 and Fig. 8 show the cluster purities for $N=300$ and $N=400$, respectively. We notice that the changing window size has minimal effect on the average cluster purity.

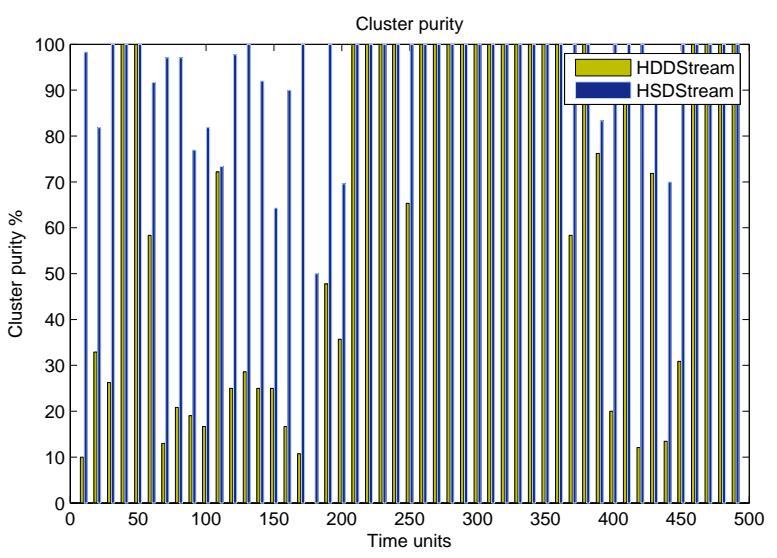

Fig. 5. Cluster purity with default values of parameters

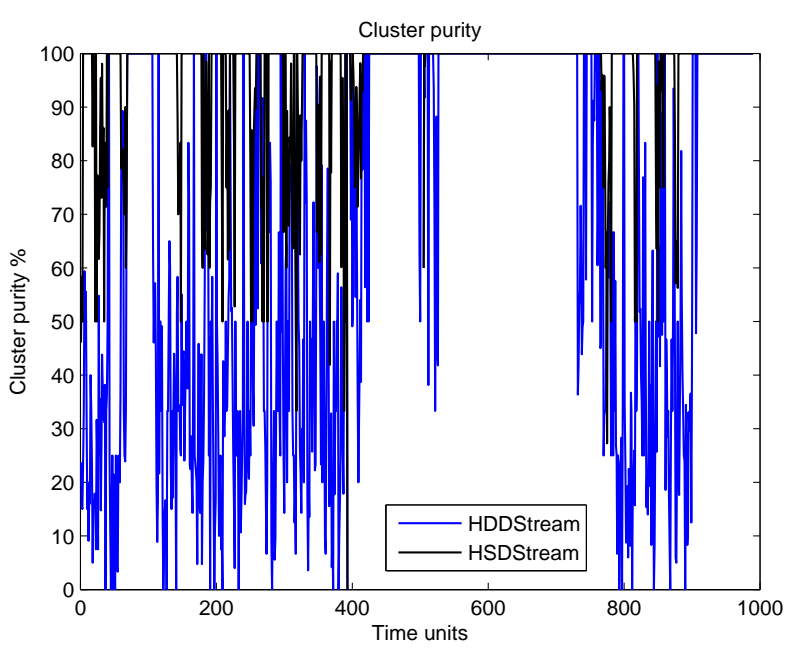

Fig. 6. Cluster purity with $N=100$

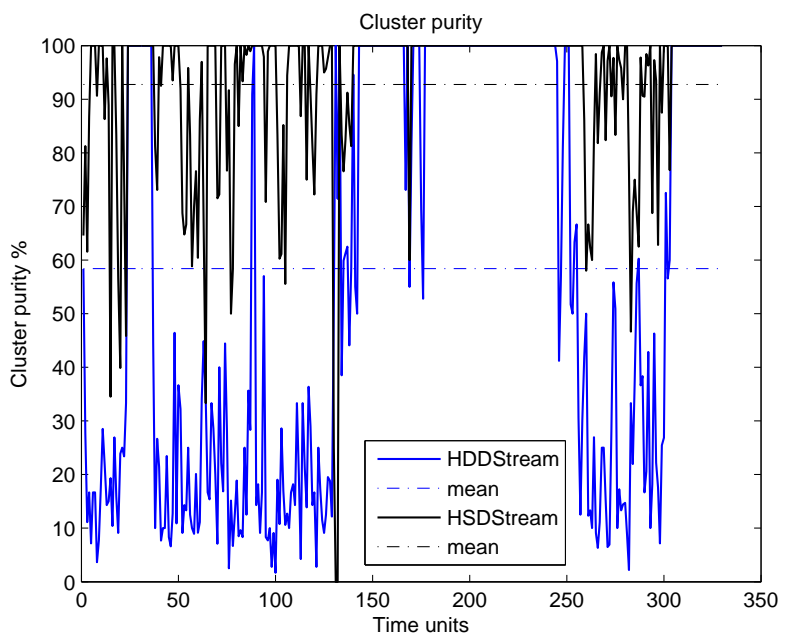

Fig. 7. Cluster purity with $N=300$

\section{Memory Usage}

We measure the memory usage as a number of microclusters in HDDStream and HDSStream. During the period 


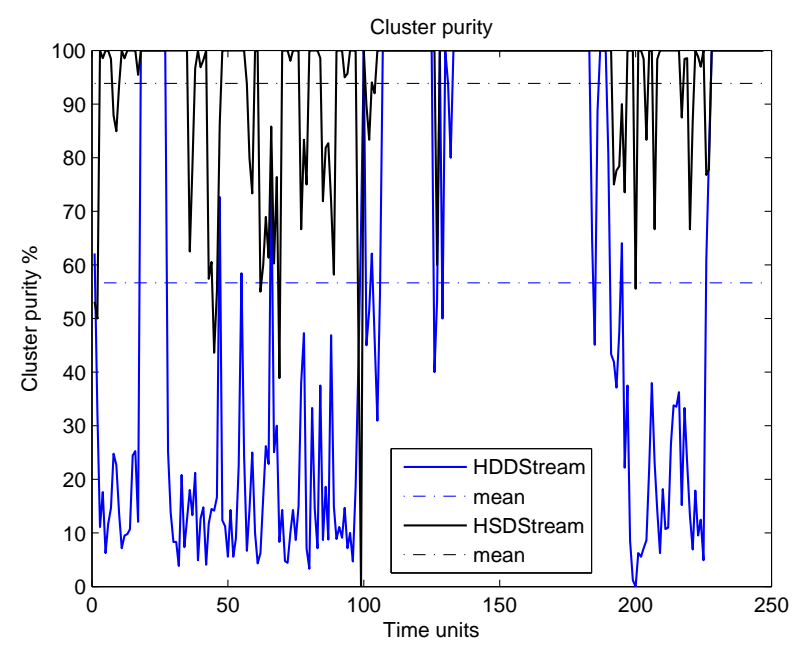

Fig. 8. Cluster purity with $N=400$

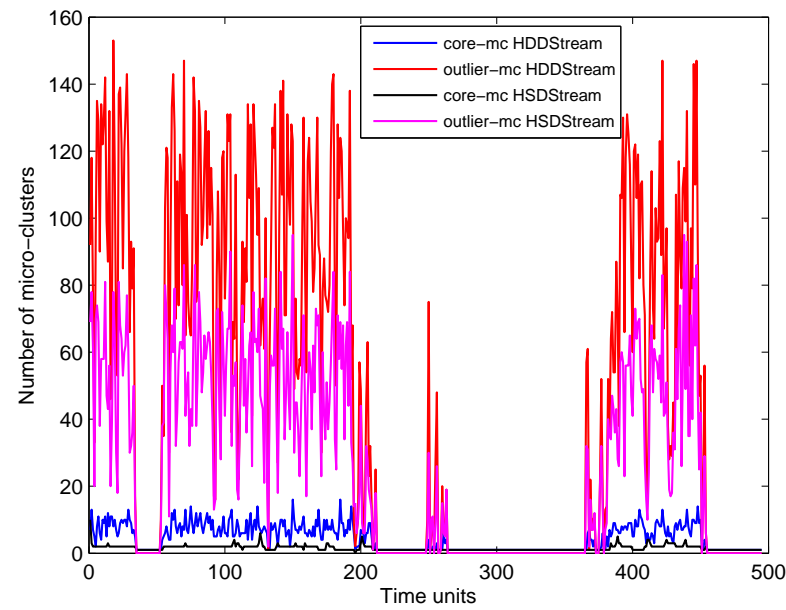

Fig. 9. Number of clusters with default values of parameters

of highly correlated normal data or the network attack, there is only one core-mc containing all the correlated points and no outlier cluster exists. It can be seen from the Figs. 10, 11,12 , and 13 that the total number of clusters is reduced to one during network attacks. When we compare these figures with different window sizes, we can see that there is a gradual increase of number of clusters with increasing number of window size. HSDStream outperforms the HDDStream in terms of memory usage for all window sizes, which is due to our reduced memory sized tuple and high density microclusters. Theoretically, the online update of $C F 1_{j}$ requires $N$ number of memory registers (one for each point's $j^{\text {th }}$ dimension), whereas, $E A 1_{j}$ needs only one memory register, as shown in Fig. 1 and Fig. 2, respectively.

\section{Sensitivity and Delay Analysis}

In sensitivity analysis, we show how sensitive the clustering quality is in relevance to the outlier threshold $\beta$, and the processing time with different window sizes. In Fig. 15 we see that cluster purity improves with increasing values of

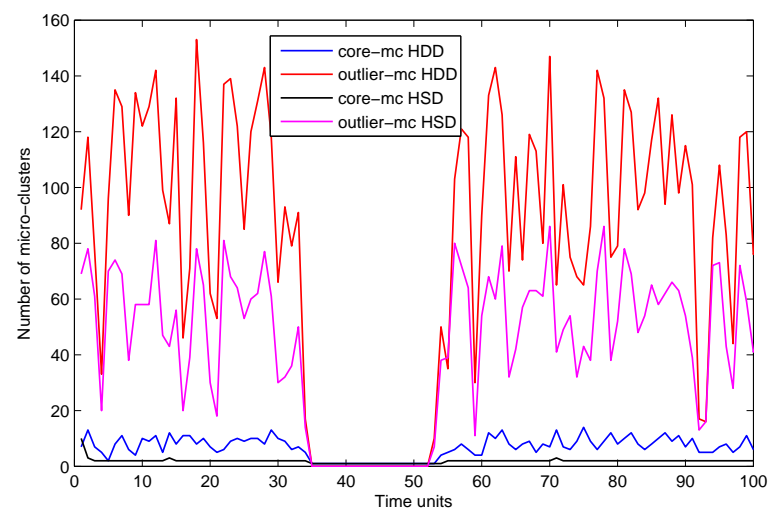

Fig. 10. Number of clusters with default values of parameters with zoom in

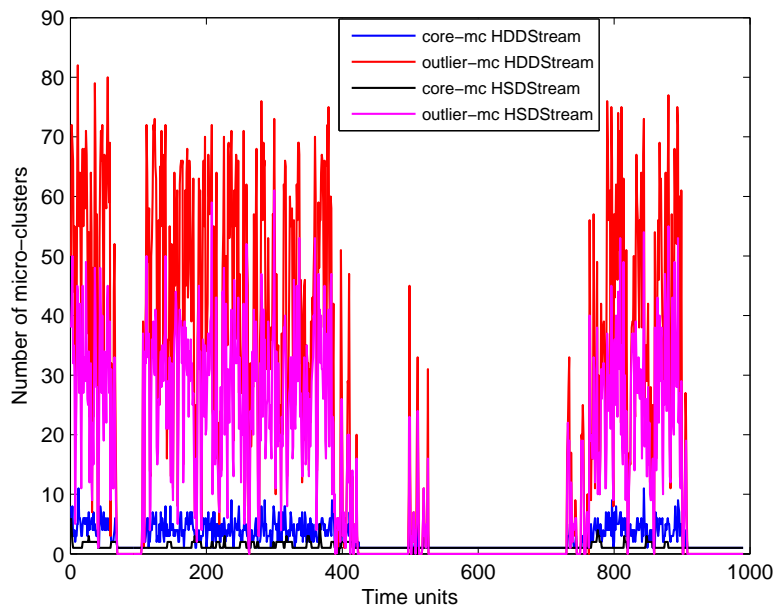

Fig. 11. Number of clusters with $N=100$

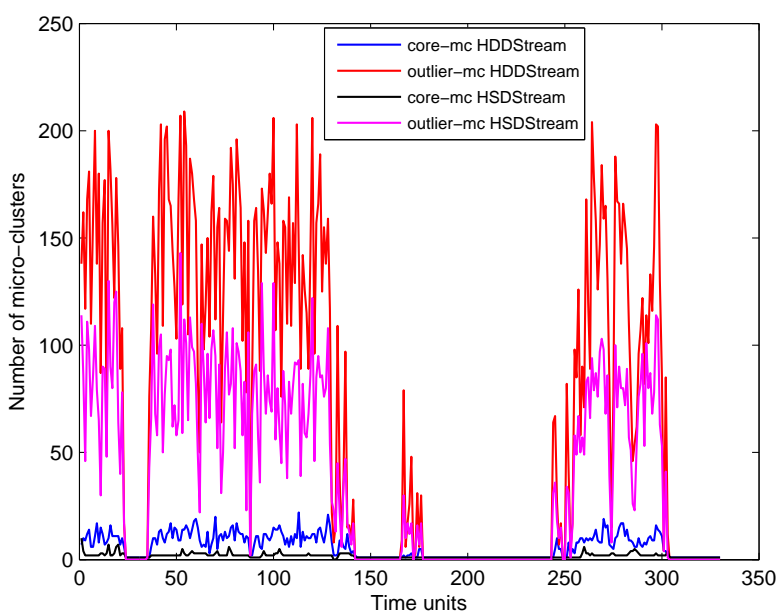

Fig. 12. Number of clusters with $N=300$

outlier threshold. Outlier threshold controls the limit of the number of points that make it eligible to become core-mc or outlier-mc. After the end of each window size, all micro- 


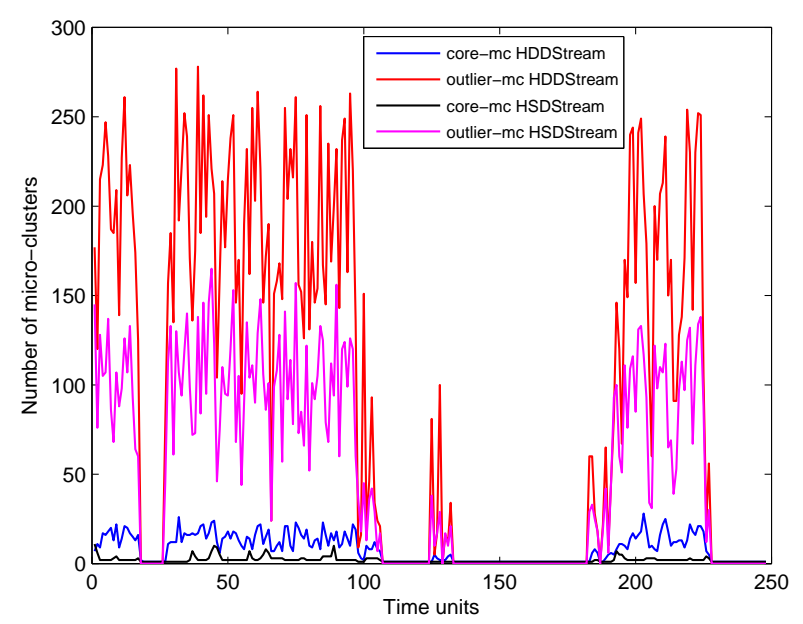

Fig. 13. Number of clusters with $N=400$

clusters are examine for their eligibility as core or outlier. For small values of $\beta$, a cluster remains its current state for the larger time duration making cluster pollute for larger duration. Whereas with high values of $\beta$ the cluster changes its state more quickly (as soon as it violate the condition NumOf Points $>$ or $<\beta \mu$ ) leaving the cluster more pure. Fig. 16 shows an important result that we can decease the memory usage by increasing the outlier threshold. Higher values of $\beta$ help remove the outlier points thus reducing the unnecessary core-mc(s). Since the core-mc(s) are small proportion of total number of clusters as shown in Fig. 10, therefore, the total number of clusters do not exhibit significant improvement in Fig. 17. However, the memory usage argument remains still valid because core-mc(s) are highly dense and utilize large proportion of memory.

Finally, we examine the processing time of HDDStream and HSDStream for different window sizes in Fig. 18. This processing time includes the time for the initialization phase and the data collection for the plotting purpose. It can be seen that HSDStream outperforms the HDDStream for all window sizes. This verifies the efficiency of our micro-cluster design in definition 1 where we need only two multipliers and one adder as compared to the conventional micro-cluster defined in definition 0 which requires $N$ number of multipliers and $N-1$ number of adders with $\left\lfloor\log _{2}(N)\right\rfloor$ stages delay. For example if $N=6$, then in order to add 6 numbers, we need 5 adders which incur 3 stages delay as shown in Fig. 14.

\section{CONCLUSION}

This paper presents a clustering algorithm for highdimensional high-density streaming data. We propose a new structure of micro-cluster's tuples. This structure uses exponential weighted averages to reduce the memory usage and decrease the computational complexity. We have compared our scheme with HDDStream with KDD network intrusion detection dataset. The results show that HSDStream give significant improvement over HDDStream in terms of cluster purity, memory usage, and the processing time.

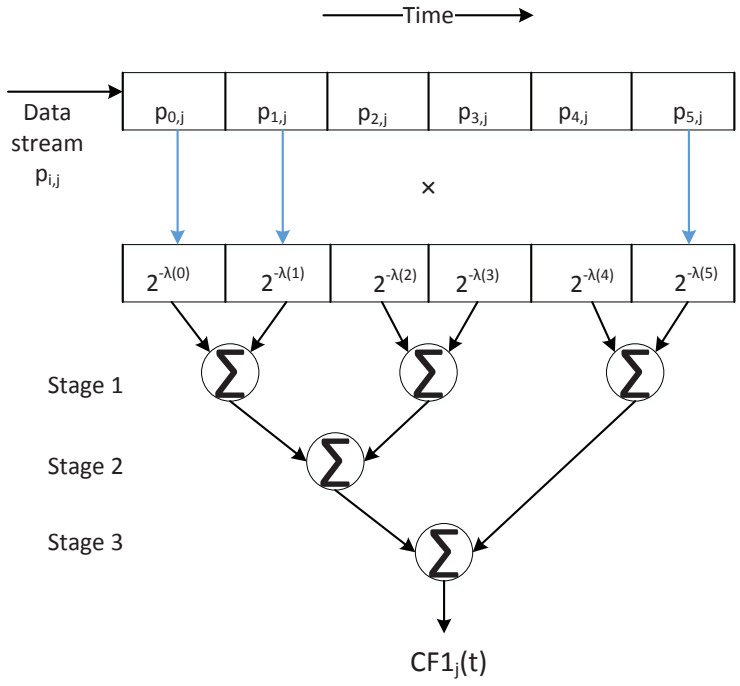

Fig. 14. Processing time delay in conventional micro-cluster update with $N=6$
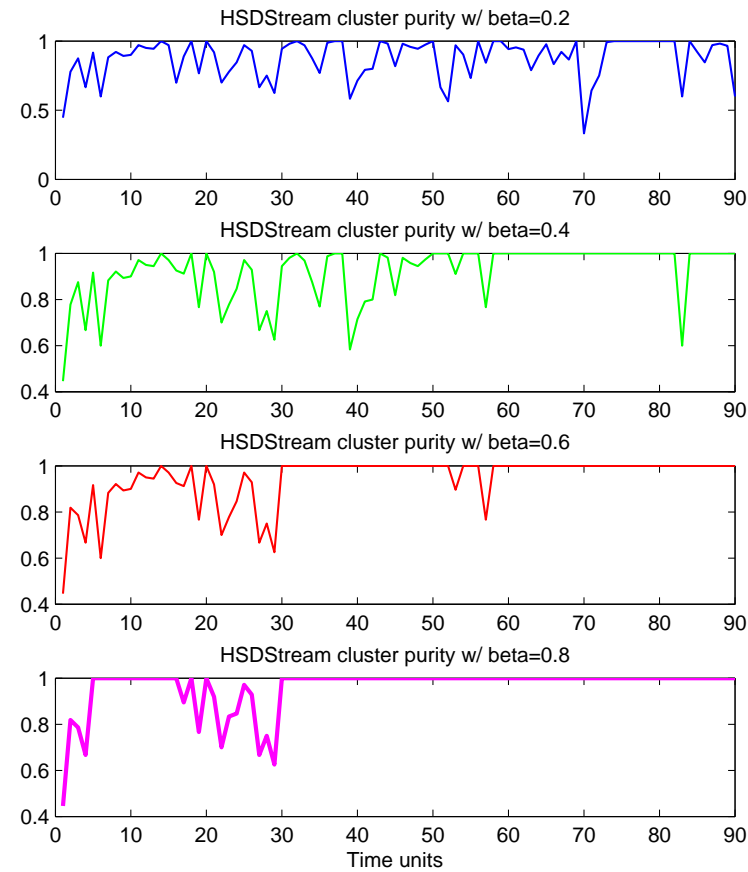

Fig. 15. Cluster purity for different values of $\beta$

\section{REFERENCES}

[1] A. Forestiero, C. Pizzuti, and G. Spezzano, "A single pass algorithm for clustering evolving data streams based on swarm intelligence," Data Mining and Knowledge Discovery, vol. 26, no. 1, pp. 1-26, Jan. 2013.

[2] K. Sim, V. Gopalkrishnan, A. Zimek, and G. Cong, "A survey on enhanced subspace clustering," Data Mining and Knowledge Discovery, vol. 26, no. 2, pp. 332-397, Mar. 2013.

[3] C. C. Aggarwal, "A segment-based framework for modeling and mining data streams," Knowledge and Information Systems, vol. 30, no. 1, pp. 

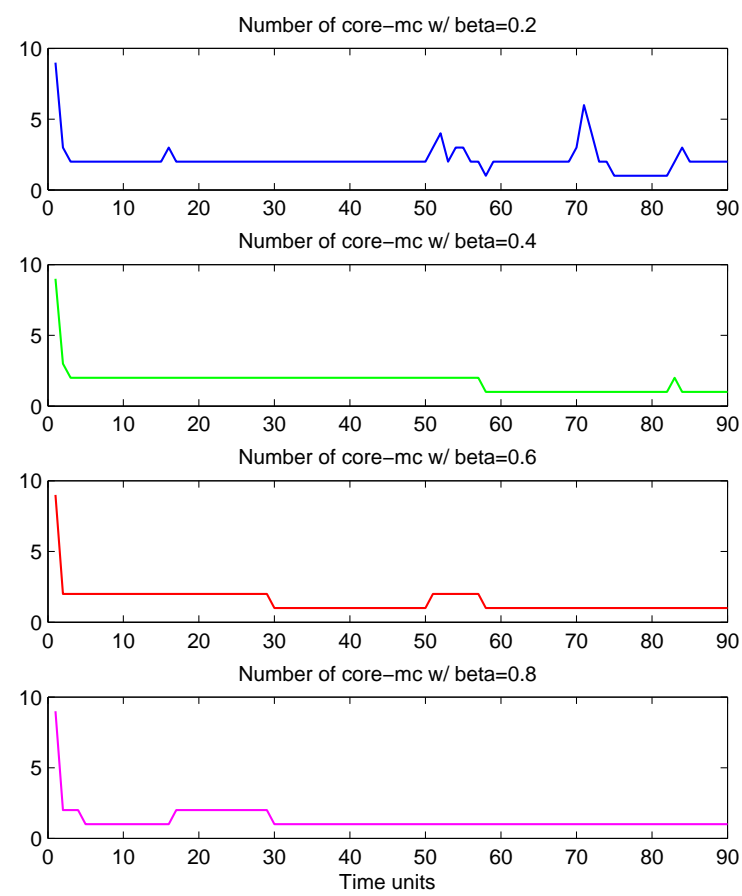

Fig. 16. Number of core-me in HSDStream versus $\beta$

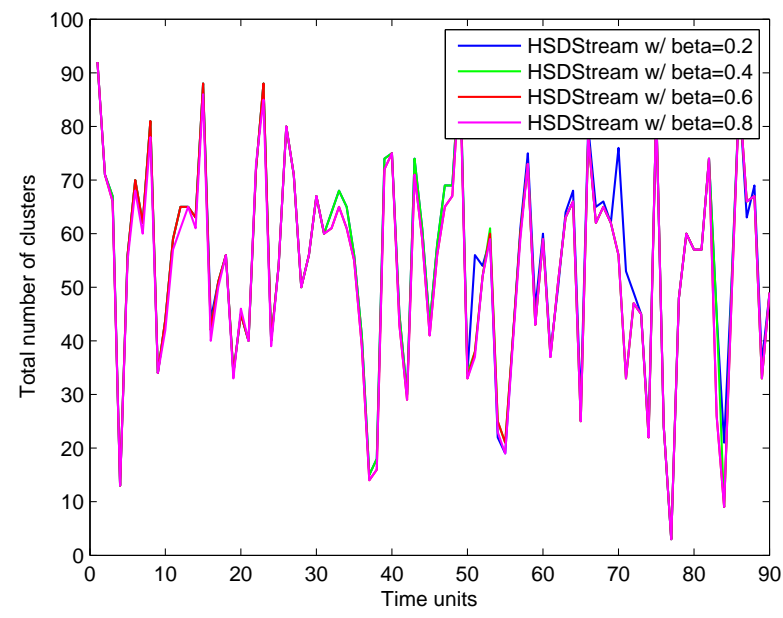

Fig. 17. Total number of clusters in HSDStream versus $\beta$

1-29, Jan. 2012

[4] A. Amini, T. Y. Wah, and H. Saboohi, "On density-based data streams clustering algorithms: A survey," Journal of Computer Science and Technology, vol. 29, no. 1, pp. 116-141, 2014.

[5] A. K. Jain, "Data clustering: 50 years beyond k-means," Pattern Recognition Letters, vol. 31, no. 8, pp. 651-666, Jun. 2010.

[6] H.-P. Kriegel, P. Krger, and A. Zimek, "Clustering high-dimensional data: A survey on subspace clustering, pattern-based clustering, and correlation clustering," ACM Trans. Knowl. Discov. Data, vol. 3, no. 1, pp. 1:1-1:58, Mar. 2009.

[7] J. MacQueen, "Some methods for classification and analysis of multivariate observations." The Regents of the University of California, 1967.

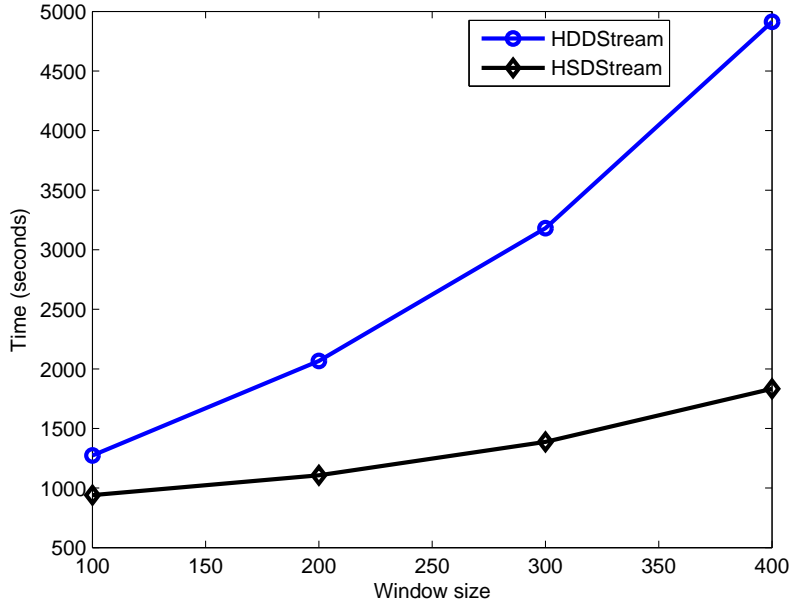

Fig. 18. Processing time for different window sizes

[8] C. C. Aggarwal, J. Han, J. Wang, and P. S. Yu, "A framework for clustering evolving data streams," in Proceedings of the 29th international conference on Very large data bases-Volume 29. VLDB Endowment, 2003, pp. 81-92.

[9] —, "A framework for projected clustering of high dimensional data streams," in Proceedings of the Thirtieth international conference on Very large data bases-Volume 30. VLDB Endowment, 2004, pp. 852863.

[10] F. Cao, M. Ester, W. Qian, and A. Zhou, "Density-based clustering over an evolving data stream with noise." in SDM, vol. 6. SIAM, 2006, pp. 326-337.

[11] I. Ntoutsi, A. Zimek, T. Palpanas, P. Krger, and H.-P. Kriegel, "Densitybased projected clustering over high dimensional data streams." in SDM. SIAM, 2012, pp. 987-998.

[12] M. Hassani, Y. Kim, S. Choi, and T. Seidl, "Subspace clustering of data streams: new algorithms and effective evaluation measures," Journal of Intelligent Information Systems, Jun. 2014.

[13] H.-L. Nguyen, Y.-K. Woon, and W.-K. Ng, "A survey on data stream clustering and classification," Knowledge and Information Systems, Dec. 2014.

[14] D. Mena-Torres and J. S. Aguilar-Ruiz, "A similarity-based approach for data stream classification," Expert Systems with Applications, vol. 41, no. 9, pp. 4224-4234, Jul. 2014.

[15] C. Jin, J. X. Yu, A. Zhou, and F. Cao, "Efficient clustering of uncertain data streams," Knowledge and Information Systems, vol. 40, no. 3, pp. 509-539, Sep. 2014.

[16] W. Liu and J. OuYang, "Clustering algorithm for high dimensional data stream over sliding windows." IEEE, Nov. 2011, pp. 1537-1542.

[17] L. Wan, W. K. Ng, X. H. Dang, P. S. Yu, and K. Zhang, "Density-based clustering of data streams at multiple resolutions," ACM Trans. Knowl. Discov. Data, vol. 3, no. 3, pp. 14:1-14:28, Jul. 2009.

[18] J. W. Lee, N. H. Park, and W. S. Lee, "Efficiently tracing clusters over high-dimensional on-line data streams," Data \& Knowledge Engineering, vol. 68, no. 3, pp. 362-379, Mar. 2009.

[19] A. Bellas, C. Bouveyron, M. Cottrell, and J. Lacaille, "Model-based clustering of high-dimensional data streams with online mixture of probabilistic PCA," Advances in Data Analysis and Classification, vol. 7, no. 3, pp. 281-300, May 2013.

[20] A. Amini, H. Saboohi, T. Herawan, and T. Y. Wah, "MuDi-stream: A multi density clustering algorithm for evolving data stream," Journal of Network and Computer Applications, Dec. 2014.

[21] R. Chairukwattana, T. Kangkachit, T. Rakthanmanon, and K. Waiyamai, "SE-stream: Dimension projection for evolution-based clustering of high dimensional data streams," in Knowledge and Systems Engineering, V. N. Huynh, T. Denoeux, D. H. Tran, A. C. Le, and S. B. Pham, 
Eds. Cham: Springer International Publishing, 2014, vol. 245, pp. 365-376.

[22] K. Waiyamai, T. Kangkachit, T. Rakthanmanon, and R. Chairukwattana, "SED-stream: Discriminative dimension selection for evolution-based clustering of high dimensional data streams," Int. J. Intell. Syst. Technol. Appl., vol. 13, no. 3, pp. 187-201, Oct. 2014.

[23] C. Bohm, K. Railing, H.-P. Kriegel, and P. Kroger, "Density connected clustering with local subspace preferences," in Fourth IEEE International Conference on Data Mining, 2004. ICDM '04, Nov. 2004, pp. 27-34.

[24] "kdd-cup-1999-computer-network-intrusion-detection."

[Online]. Available: http://www.sigkdd.org/

kdd-cup-1999-computer-network-intrusion-detection 